\title{
Understanding chinese medicine and western medicine to reach the maximum treatment benefit
}

\author{
Cao $C^{1,2,3 *}$ and Brown $B^{1}$ \\ ${ }^{1}$ College of Pharmacy, University of South Florida, USA \\ ${ }^{2}$ College of Medicine, University of South Florida, USA \\ ${ }^{3}$ College of Arts \& Sciences, University of South Florida, USA
}

Medicine can be traced back to as early as the origin of man, since food and medicine are intertwined. Some foods can be used for medicinal purposes, and some foods with medicinal properties are also used as everyday foods, such as spices used in cooking around the world. This is very much true of herbal medicine. When we are healthy, these medicinal foods can be consumed without restriction such as in daily eating, however when we are sick, the medicinal foods or edible materials are restricted and used as a drug in the traditional sense where the quantity used or consumed is regulated.

When we moved towards industrialization, modern Western medicine became the dominant medical practices with penicillin as a key discovery in disease treatment and exploration. Since then, herbal medicine gradually lost its dominant position in disease treatment. With this significant achievement in treating infectious disease, more and more molecules have been synthesized for treating various known diseases. Life expectancy has since increased significantly, and we have been able to fight infectious and chronic diseases with newer, targeted medications [1-3]. With this increased life expectancy, we are facing new diseases associated with aging, and scientists have discovered that these single molecule drugs are no longer able to effectively treat more complicated conditions $[2,4,5]$. A new way of dealing with age-related health issues is urgently needed, and so understanding the differences between herbal medicine and Western medicine can help us face this growing challenge.

We are challenged by the choice of using traditional medicine or modern medicine. Traditional medicine, known as Traditional Chinese medicine, includes surgery, moxibustion, hot cupping, acupuncture, massage, herbal medicine and nutraceutical medicine. Modern medicine, known as Western medicine, includes surgery and most commonly single molecular drugs. Here, we want to share our opinion with readers on these two approaches to better assist with choosing the proper method to treat a disease that we are targeting.

The major differences between western medicine and chinese medicine

Western medicine aims to eliminate an existing problem.

a. Chinese medicine diagnoses through the symptoms of the patient described and appearances (eye, skin and tongue color as well as pulse), then seeks to address the overall systemic problem with a focus on preventing any potential adverse effects [6].

b. Western medicine treats symptoms and treats the target or target organ as isolated from the rest of the body instead of as one whole interconnected system. Western medicine provides diagnosis through lab test and it focuses on eliminating symptoms but normally fails to address adverse effects on the body.

Chinese medicine focuses on the body's overall response to treatment and recognizes the body as one interconnected biosystem. Treatment changes the overall condition of the body including the immune system, but also takes care of the specific target problem.

\section{Onset time of drugs}

a. Western medicine typically has rapid or immediate effects, so it is highly effective for life-threatening conditions, such as infectious diseases. However, the major issue associated with these drugs is the potential damage they may have to other parts of body even though it is a life-saving procedure or method. Some modern drugs can leave potential permanent damage to other parts of the body. For example, antibiotic treatment with tetracycline can cause adverse effects that include permanent staining of the teeth.

b. Chinese medicine is designed to both prevent adverse effects and treat diseases. The onset of drug efficacy compared to Western medicine is longer, but it is safer to use, since it takes into account potential adverse effects. It can also prevent the secondary or aftereffects caused by treatments seen in Western medicine.

\section{Benefit duration}

a. Chinese medicine can have effects or changes in the overall immune system, so it can prevent future issues or reoccurrence of an illness that Western medicine is unable to address. Thus, Chinese medicine addresses the root cause of the disease whereas Western medicine sometimes can only address the symptoms.

b. Western medicine takes effect immediately but also loses its function quickly due to their rapid metabolism, thus it has no long-term benefits but may cause long term adverse effects.

\section{The differences in mechanism}

a. Western medicine focuses primarily on the use of single molecular drugs which makes it easier to identify targeted molecules and related signal pathways in research. Therefore, most drugs developed rely on chemical synthesis.

${ }^{*}$ Correspondence to: Chuanhai Cao Ph.D, Department of Pharmaceutical Sciences, College of Pharmacy, University of South Florida MDC4106A 12901 Bruce B. Downs Blvd, Tampa FL 33612, USA, E-mail: ccao@health.usf.edu

Received: March 01, 2019; Accepted: April 19, 2019; Published: May 03, 2019 
b. Chinese medicine tries to address the biosystem as a whole instead of using single molecules on single targets. The aim is to change the bioenvironment of the body (overall immune system) to achieve long-lasting effects. Normally, Components in Chinese medicine work as one formula, so they are inseparable [7].

\section{Common goals between western and chinese medicine}

a. Both Chinese medicine and Western medicine are aimed at curing the underlying cause of the disease. However, many differences exist between their approaches.

b. Chinese medicine and Western medicine may target the same molecule or same pathways to cure the diseases. Therefore, they may have similar effects in treating certain diseases.

\section{Maximum treatment benefits combining western and chinese medicine}

a. It is not inherently good or bad to use one method or the other to treat disease. We should combine Western and Chinese medicine. The best approach is a combination therapy using Western medicine to alleviate current symptoms and using Chinese medicine concurrently to address the root cause of the disease as well as preventing disease reoccurrence. This method uses Western medicine as a key element, and the Chinese medicinal approach serves as an assistant addresser and messenger.

b. There is a principle of traditional medicine called "Jun-Chen-ZuoShi" which covers four functions:

1. Denominator or key element: A drug or a molecule that directly fights or targets the pathological factor related to a disease (ie. an interferon or anti-virial drug that can kill tumor cells or viruses).

2. Assistant or Enhancer: A drug or a molecule that can enhance the function of the drug (ie. An adjuvant which can enhance the function of an antigen).

3. Corrector or addresser of adverse effects (limiting factor): A drug or a molecule that can prevent adverse effects that are related to the denominator or enhancer in order to limit abnormal responses.

4. Messenger: A drug or a molecule that can bring the function of the denominator to the target site.

Target effects or precision medicine and personal medicine

a. Western medicine takes effect immediately but can have difficulties reaching the target or target organ. Chinese medicine is a personalized and precision medicine $[8,9]$. whereas Western medicine is a single target medicine. Given this, they should be combined. The Western medicine approach is a singular part of Chinese medicine.

b. Chinese medicine is a personalized and precision medicine. First, the drug formula needs to be tailored based upon the response and needs of the patient which are based on an evaluation of the patient's symptoms, such as tongue and skin color etc.). Secondly, Chinese medicine adds or removes certain ingredients upon further examination of a patient's response to the treatment, so it is a personalized. Also, Chinese Medicine can have different formula for the same disease upon the patient's symptom, therefore it is a precision medicine [10]. However, Western medicine is typically in fixed doses of specific medications, such as pill or capsule, so it is not designed for personal or precision medicine.

\section{The connection between western and chinese medicine}

a. Western medicine has used our immune system as a diagnostic tool, including immunotyping and tracking immune responses (adopted immunity or acquired immunity, Th1 or Th2, autoimmune response or immunotolerance). Chinese medicine uses "Yin and Yang" or "Kidney deficiency or spleen deficiency" as key features to evaluate body responses to treatment.

b. The common connection between Western and Chinese medicine is the immune system. Western medicine can use the immune system to do personalized and precision treatment, such as cancer [11], and Chinese medicine is designed and practiced as personalized and precision medicine [12]. Through the quantification to Kidney and Spleen deficiency, we may find the mislinkage between Chinese medicine and western medicine, and then find a best treatment method for a disease.

\section{Conclusion}

We believe the best treatment approach to use to treat human disease should follow the principle of Chinese medicine "Jun-ChenZhou-Shi" and combine it with Western medicine (Jun) and combine all other elements in the formula in order to prevent adverse effects [13].

\section{References}

1. Doblhammer G, Kreft D, Dethloff A (2012) Life years gained. Long-term trends in mortality by causes of death in Germany and in an international perspective. Bundesgesundheitsblatt Gesundheitsforschung Gesundheitsschutz 55: 448-58.

2. Yang G, Kong L, Zhao W, Wan X, Zhai Y, et al. (2008) Emergence of chronic noncommunicable diseases in China. Lancet 372: 1697-1705. [Crossref]

3. Huicho L (2009) Mortality profiles in a country facing epidemiological transition: An analysis of registered data. BMC Public Health 9: 47.

4. Paccaud F (2002) Rejuvenating health systems for aging communities. Aging Clin Exp Res 14: 314-318. [Crossref]

5. Patrick WK, Cadman EC (2002) Changing emphases in public health and medica education in health care reform. Asia Pac J Public Health 14: 35-39. [Crossref]

6. van der Greef J (2010) Systems biology-based diagnostic principles as pillars of the bridge between Chinese and Western medicine. Planta medica 76: 2036-2047.

7. Qiu J (2007) Traditional medicine: A culture in the balance. Nature 448: 126-128.

8. Li L (2019) The role of chinese medicine in health maintenance and disease prevention: Application of constitution theory. Am J Chin Med 9: 1-12.

9. Schleidgen $\mathrm{S}$ (2013) What is personalized medicine: Sharpening a vague term based on a systematic literature review. BMC Medical Ethics 11: 55.

10. Sun XG, Wu Wk, Lu Zp (2012) Chinese integrative medicine: Translation toward person-centered and balanced medicine. Chinese journal of integrative medicine 18: 3-6.

11. Paucek RD, Baltimore D, Li G (2019) The cellular immunotherapy revolution: Arming the immune system for precision therapy. Trends Immunol 40: 292-309. [Crossref]

12. Wang S, Long S, Wu W (2018) Application of traditional chinese medicines as personalized therapy in human cancers. Am J Chin Med 46: 953-970. [Crossref]

13. Jafari S, Abdollahi M, Saeidnia S (2014) Personalized medicine: A confluence of traditional and contemporary medicine. Alternative Therapies in Health \& Medicine 20: 31-40. [Crossref]

Copyright: (C2019 Cao C. This is an open-access article distributed under the terms of the Creative Commons Attribution License, which permits unrestricted use, distribution, and reproduction in any medium, provided the original author and source are credited. 